Planetary Systems in the Universe - Observation, Formation and Evolution

Proceedings IAU Symposium No. 202, (C2004 IAU

Alan Penny, Pawel Artymowicz, Anne-Marie Lagrange, $\&$ Sara Russell, eds.

\title{
STIS Coronagraphic Observations of $\beta$ Pictoris
}

\author{
S. R. Heap, D. J. Lindler and T. M. Lanz \\ Laboratory for Astronomy $\&$ Solar Physics \\ NASA's Goddard Space Flight Center, Greenbelt, MD 20771, USA
}

\begin{abstract}
We present recent coronagraphic observations of $\beta$ Pictoris obtained with the Space Telescope Imaging Spectrograph (STIS) aboard the Hubble Space Telescope. The superb, high-resolution images show that the inner part of the disk is inclined by about $5^{\circ}$ with respect to the main disk. Longslit coronagraphic spectrograms oriented along the inner disk indicate that the reflectance of the inner disk is neutral over the spectral region, 3000-5600 $\AA$.
\end{abstract}

\section{Introduction}

$\beta$ Pictoris is an intensively studied Vega-type system composed of a relatively young $\left(<10^{8} \mathrm{yr}\right) \mathrm{A} 5 \mathrm{~V}$-type star surrounded by a large dust disk, which is seen edge-on. As one of the closest analogues to the early Solar System (Artymowicz 1997, Lagrange et al. 2000), it merits close scrutiny and analysis. Here, we report on coronagraphic observations of $\beta$ Pic made with the Space Telescope Imaging Spectrograph (STIS) aboard the Hubble Space Telescope. Because of its high resolution $\left(0^{\prime \prime} 1=1.93 \mathrm{AU}\right.$ at the Hipparcos distance of $\beta$ Pic), STIS coronagraphic observations define the structure of the disk with greater precision and at closer radii to the star than could previous observations.

\section{Coronagraphic Imagery}

We obtained white-light coronagraphic images of $\beta$ Pic using an occulting mask either $1^{\prime \prime}$ or $2^{\prime \prime}$ wide. The observational results are described by Heap et al. (2000). The STIS images define the structure of the disk with high precision and at close projected radii (15 AU). Figure 1 shows a contour plot of $\beta$ Pic with contours at brightness levels equal to $0.1,0.5$, and 0.99 times the maximum brightness at a given projected radius. The spine of the disk ( $99 \%$ contours) has a near-constant thickness but is curved. At half-maximum brightness, the disk shows bulges on either side. It is these bulges at $r=60-90 \mathrm{AU}$ that give the disk its warped appearance. Only at the $10 \%$ contours does the disk take on a wedge shape.

Measurements of the vertical profile of the disk indicate that the warped disk can be resolved into two components: a main component having a position angle, $\mathrm{PA}=30^{\circ}$; and a fainter component inclined by $5^{\circ}\left(\mathrm{PA}=35^{\circ}\right)$ which is visible out to only $\sim 100 \mathrm{AU}$. The apparent bulges are actually extensions of the tilted component above and below the main component. The fainter, tilted disk is similar to MIR-emitting disk observed by Pantin et al. (1997) in having the 


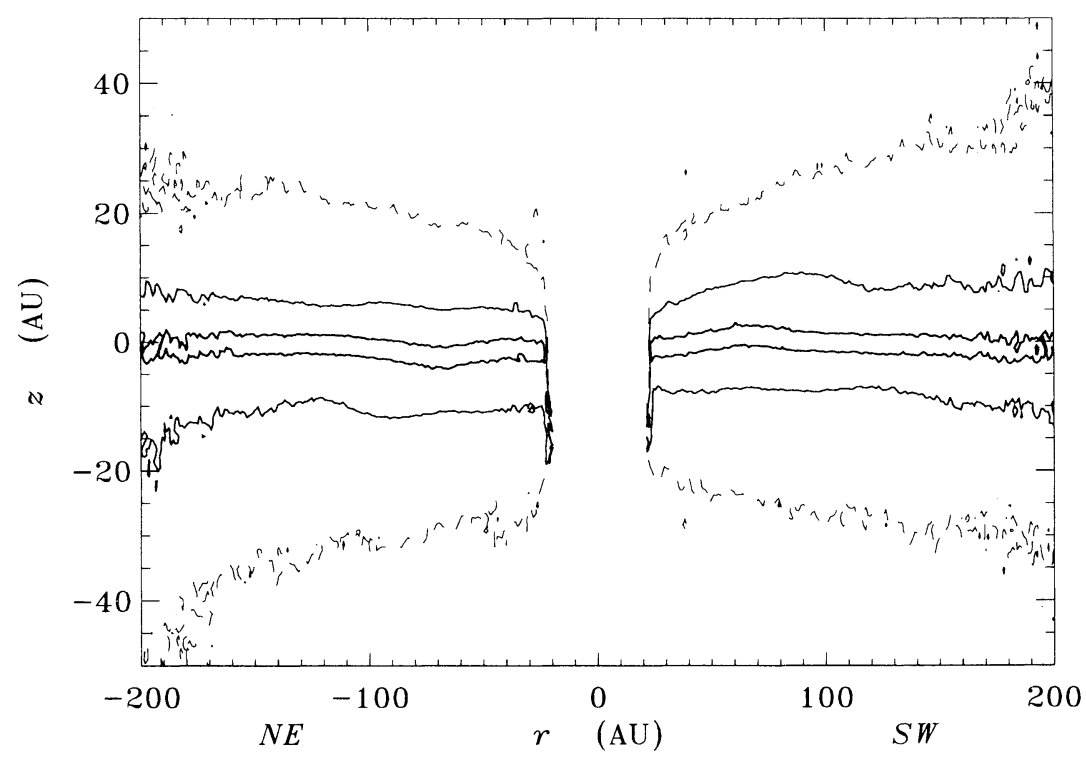

Figure 1. $\quad$ Contour plot of the $\beta$ Pic disk. Three brightness levels are shown: the spine of the disks ( $\geq 99 \%$ of the maximum brightness), the FWHM brightness, and $10 \%$ maximum brightness.

same orientation $\left(\right.$ P.A. $=35^{\circ}$ ) and similar radial brightness distributions. These similarities imply that the fainter, tilted component arises in the inner region of the disk, and the main component, in the outer disk.

Following Burrows et al. (1995) and Mouillet et al. (1997), Heap et al. (2000) interpreted the tilt of the inner disk as evidence of gravitational perturbation by a planet in a slightly inclined orbit. They used the analytic scaling relations of Larwood \& Papaloizou (1997) to derive a planetary mass of 0.17 Jupiters if the age of the system is $20 \mathrm{Myr}$, and the orbital radius of the planet is $50 \mathrm{AU}$. These results have now been superceded by more detailed analysis and modeling by Gorkavyi et al. (2000) presented at this conference

\section{Coronagraphic Spectroscopy}

There is both theoretical and observational evidence that the dust composition of the inner (warmer) disk of beta Pic differs from that of the outer disk (c.f. Artymowicz 2000). One of the most intriguing observations on this topic are those made by Ferlet et al. (1994), who conducted BVRI photometry of the disk using an anti-blooming CCD. Their photometry indicated that in the outer disk $\left(r=7^{\prime \prime} .5\right)$, the disk has the same flux distribution as the star, but closer in to the star $(r=2 ! 5)$, the dust reflectance at the B filter drops down to about $20 \%$ of its outer value. Such results are important in posing constraints on the 


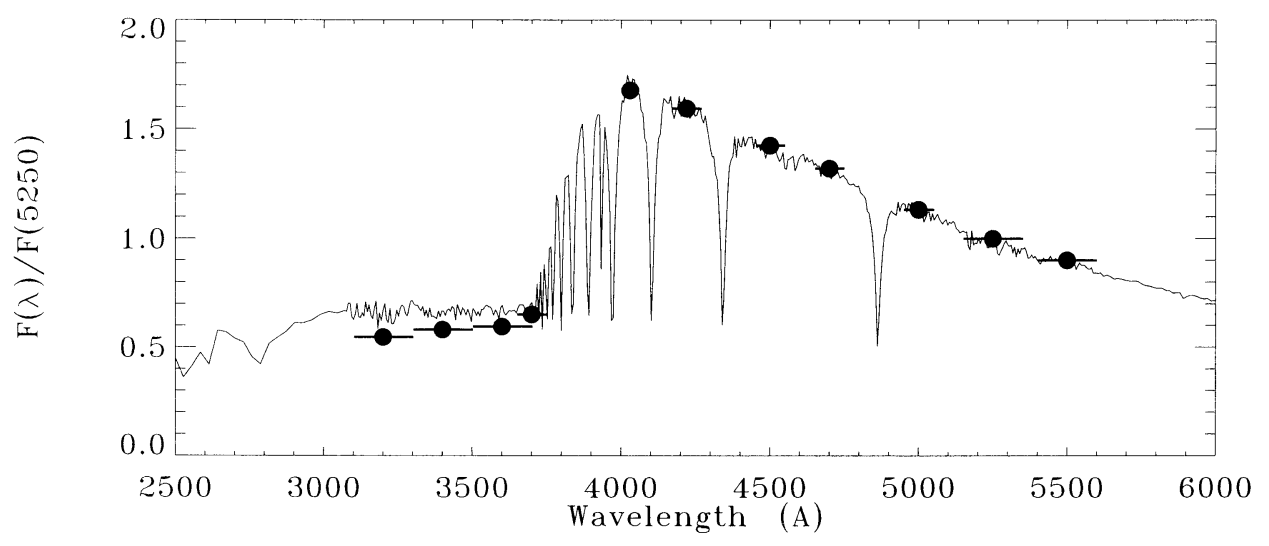

Figure 2. $\quad$ Spectral energy distributions of the star (solid line) and disk (filled circles) in the inner, tilted disk at $r=4^{\prime \prime}$.

dust composition via comparison with dust reflectance spectra measured in the laboratory.

STIS coronagraphic spectroscopy offers the best opportunity to sample the reflectance spectrum of the inner disk. We therefore obtained a long-slit spectrogram of the $\beta$ Pic disk with a 0 "' 5 -wide slit oriented along the inner, tilted disk $\left(\mathrm{PA}=35^{\circ}\right)$, and its occulting bar blocking the star. Figure 2 shows the resulting brightness distribution after removal of the diffracted + scattered light from the star. At $r=44^{\prime \prime} 0$, where the inner disk extends furthest from the spine of the main disk, the relative flux distributions of the disk and star are nearly identical. It is possible that this sample is still contaminated by contributions from the outer disk. In any case, we are forced to conclude that diagnostic spectral features or cutoffs in the reflectance spectrum of the inner disk $(r<90 \mathrm{AU})$ still elude us.

\section{References}

Artymowicz, P. 1997, Ann. Rev. Earth Planetary Sci., 25, 175

Artymowicz, P. 2000, Space Science Reviews, 92, 69

Burrows, C., Krist, J.E., Stapelfeldt, K. 1995, BAAS, 187 \#32.05

Ferlet, A., Lecavelier des Etangs, A., et al. 1994, Ap\&SS, 212, 173

Heap, S.R., Lindler, D.J., Lanz, T.M, et al. 2000, ApJ, 539, 4351

Mouillet, D., Larwood, J., Papaloizou, J., Lagrange, A.M. 1997, MNRAS, 292, 896 